\title{
CARACTERIZAÇÃO AGROMORFOLÓGICA DO MARACUJAZEIRO (Passiflora spp.) ${ }^{1}$
}

\author{
MARIA LÚCIA CROCHEMORE², HUGO BRUNO MOLINARI ${ }^{2}$, NEUSA MARIA COLAUTO STENZEL ${ }^{2}$
}

\begin{abstract}
RESUMO - Esse estudo descreve a variação genética de 55 acessos de Passiflora spp., constituídos de P. edulis, P. alata, P. coccinea, $P$. caerulea, $P$. foetida, P. giberti, P. macrocarpa, P. macrocarpa $x$ alata, P. serrato digitata, . suberosa e um acesso Passiflora sp. Vinte e dois descritores morfológicos foram avaliados sobre plantas isoladas em sistema de espaldeira e permitiram estruturar a diversidade encontrada. As relações filogenéticas entre os acessos, avaliadas pela análise de componentes principais e de distâncias genéticas, mostraram existir ampla diversidade entre as espécies estudadas. Algumas espécies mostraram caracteres monomórficos. Importante variabilidade foi observada dentro de $P$. alata e de $P$. edulis, e pequenas divergências foram encontradas entre os acessos da forma flavicarpa.
\end{abstract}

Termos para indexação: diversidade, descritores, morfologia, botânica.

\section{AGRO-MORPHOLOGICAL CHARACTERIZATION OF PASSION FRUIT (Passiflora spp.) GERMOPLASM}

ABSTRACT - The germoplasm evaluated is composed by 55 accessions of $P$. edulis, $P$. alata, P. coccinea, $P$. caerulea, $P$. foetida, $P$. giberti, P. macrocarpa, P. macrocarpa x P. alata, P. serrato digitata and P. suberosa; and one Passiflora sp. accession. On isolated plants field cultivated under trellising system, 22 morphological descriptors were studied. The phylogenetic relationships evaluated by the Principal Component Analysis and genetic distances showed a wide genetic diversity among the species studied. Monomorphics characters were showed by some species. Important variability is observed within P. alata and P. edulis and small divergences are found between accessions of the flavicarpa form.

Index terms: diversity, descriptors, morphology.

\section{INTRODUÇÃO}

O gênero Passiflora é originário da América do Sul e tem no Centro-Norte do Brasil, o maior centro de distribuição geográfica (Lopes, 1991). Entre as 530 espécies descritas para o gênero, mais de 150 são nativas do Brasil (Hoehne, 1946). Somente algumas têm importância econômica em função da qualidade dos frutos para consumo ou ainda por apresentarem propriedades medicinais. Entretanto, os cultivos comerciais de maracujazeiro compreendem basicamente o maracujazeiro-roxo (P. edulis Sims.) e o maracujazeiro-amarelo ( $P$. edulis f. flavicarpa Degener.). O maracujazeiro-roxo é largamente cultivado em diversos países do mundo, enquanto o amarelo é o mais cultivado no Brasil, sendo atualmente o maior produtor mundial. Estima-se que haja dois números cromossômicos básicos no gênero Passiflora: $\mathrm{x}=6 \mathrm{e} \mathrm{x}=9$. Apenas 75 espécies têm o número cromossômico estabelecido. Quanto ao nível de ploidia, a maioria das espécies são diplóides ( 2 n=12 ou 18), existindo alta compatibilidade interespecífica em cruzamentos dentro desse grupo (Bruckner, 1997). P. edulis tem $2 \mathrm{n}=18$ cromossomos

Os estudos taxonômicos em Passiflora baseiam-se na caracterização morfológica e agronômica da planta, levando a uma classificação nítida até o táxon espécie. Porém, dentro das espécies, as dissimilaridades existentes apresentam maiores dificuldades para serem observadas e caracterizadas. Normalmente, em Passiflora edulis, a caracterização está relacionada ao fruto e a algumas características pomológicas, tais como produção/safra, peso do fruto, tamanho do fruto, rendimento do suco, brix e acidez do suco, porém estes caracteres quantitativos não são precisos sob o plano taxonômico. Para Martin \& Nakasone (1970), além da coloração do fruto, sabor e resistência às doenças, $P$. edulis e $P$. edulis f. flavicarpa não apresentam diferenças contrastantes entre si.

O banco de germoplasma estudado compreende acessos originários das mais diversas regiões geográficas e de várias introduções e seleções da forma flavicarpa de $P$. edulis, que ainda não foram totalmente caracterizados. Esse estudo tratou, portanto, da caracterização de 55 acessos (populações, variedades, linhagens, híbridos) de Passiflora spp., por meio de 22 descritores agromorfológicos da planta e permitiu uma aproximação da estruturação da diversidade existente entre as espécies e, principal- mente, dentro de P. edulis.

\section{MATERIAL E MÉTODOS}

O material estudado incluiu 8 acessos de $P$. edulis Sims., 34 acessos de $P$. edulis Sims f. flavicarpa Degener, 3 acessos de $P$. alata Dryand, um acesso de cada de uma das seguintes espécies e/ou híbridos: P. coccinea Aubl., P. caerulea Linn., P. foetida Linn., P. giberti N.E. Brown., P. macrocarpa Mast., P. macrocarpa $x$ alata, $P$. serrato digitata, $P$. suberosa Linn., P. edulis $x$ P. edulis f. flavicarpa e um acesso Passiflora sp. A identificação das espécies e a origem geográfica dos acessos estão apresentadas na Tabela 1 .

$\mathrm{O}$ experimento foi realizado sobre plantas isoladas de uma coleção de germoplasma de maracujazeiro instaladas em campo, em sistema de espaldeira, espaçadas de $3 \times 3 \mathrm{~m}$, com dois fios de arame, na Estação Experimental do IAPAR, em Londrina - Paraná - Brasil (long $51^{\circ} 11^{\prime} \mathrm{W}$, lat $23^{\circ} 23^{\prime} \mathrm{S}$, alt $566 \mathrm{~m}$ ). As avaliações morfológicas envolveram descritores da folha, estípula, haste, gavinha e fruto de plantas com seis meses de desenvolvimento e foram realizadas no período compreendido entre março e junho de 2001: FF = Forma da Folha; $\mathrm{DH}=$ Diâmetro da Haste; $\mathrm{FE}=$ Forma da Estípula; $\mathrm{CAF}=$ Comprimento/Altura Folha; $\mathrm{PTF}=$ Presença de Tricomas na Folha; $\mathrm{CLF}=$ Comprimento do Lóbulo da Folha; PAH = Presença de Antocianina nas Hastes; $\mathrm{CEG}=$ Comprimento do Espiral Gavinha; $\mathrm{PAG}=$ Presença de Antocianina na Gavinha; DEG = Diâmetro do Espiral Gavinha; PAE = Presença de Antocianina nas Estípulas; $\mathrm{CPF}$ $=$ Comprimento do Pecíolo Folha; $\mathrm{FH}=$ Formato de Haste; $\mathrm{CIH}=$ Comprimento do Internódio Haste; $\mathrm{MF}=$ Margem Folha; $\mathrm{CBPEG}=$ Comprimento da Base à Ponta do Espiral Gavinha; $\mathrm{CFL}=\mathrm{Cor}$ da Flor; GFP $=$ Número de Glândulas Foliares e Peciolares; $\mathrm{CCF}=$ Cor da Casca do Fruto; LTF = Largura Total da Folha; COP = Cor da Polpa; FOF $=$ Formato do Fruto. Os descritores, códigos: e as unidades utilizados na caracterização encontram-se listados na Tabela 2.

Tendo em vista a necessidade de obter-se caracterização dos acessos em função de um conjunto de variáveis simultaneamente analisadas, procedeu-se a uma análise multivariada do tipo Componentes Principais - ACP. Para representar as relações entre os acessos e caracterizar a diversidade, distâncias Euclidianas foram calculadas sobre as variáveis, as quais permitiram a construção de 
dendrogramas pelo algorítimo de classificação hierárquica ascendente UPGMA (Unweighted Pair Grouped Method Average). Todas as aná- lises foram realizadas com o Programa estatístico SAS, e os gráficos foram obtidos com o Programa Statistica for Windows.

TABELA 1- Códigos, espécies e origem dos acessos da coleção de germoplasma de Passiflora spp. usados para avaliar a diversidade genética.

\begin{tabular}{|c|c|c|c|c|c|}
\hline Acesso Código & Espécie & Origem & Acesso Código & Espécie & Origem \\
\hline BG01 & Passiflora macrocarpa & Brasil-SP & LM 8 & P. edulis f. flavicarpa & Brasil-PR \\
\hline BG04 & Passiflora edulis & Quênia & LM9 & P. edulis $\mathrm{f}$. flavicarpa & Brasil-PR \\
\hline BG05 & P. alata $\times$ P. macrocarpa & Brasil - SP & LM 10 & P. edulis $\mathrm{f}$. flavicarpa & Brasil-PR \\
\hline BG06 & Passiflora alata & Brasil & IA 80 & P. edulis f. flavicarpa & Brasil-MG \\
\hline BG08 & P. serrato digitata & Brasil - SP & IA 65 & P. edulis $\mathrm{f}$ flavicarpa & Brasil-MG \\
\hline BG09 & Passiflora edulis & Brasil - SP & IA 47 & P. edulis f. flavicarpa & Brasil-MG \\
\hline BG 10 & Passiflora caerulea & Brasil - SP & IA 46 & P. edulis $\mathrm{f}$. flavicarpa & Brasil-PR \\
\hline BG11 & Passiflora suberosa & Brasil - SP & IA 42 & P. edulis f. flavicarpa & Brasil-MG \\
\hline BG 14 & Passiflora edulis & Brasil - SP & IA 31 & P. edulis $\mathrm{f}$. flavicarpa & Brasil-MG \\
\hline BG15 & Passiflora coccinea & Brasil - AM & IA 30 & P. edulis f. flavicarpa & Brasil-SP \\
\hline BG17 & Passiflora giberti & Brasil - SP & IA 27 & P. edulis $f$. flavicarpa & Brasil-SP \\
\hline BG18 & P. edulis $\times$ P. edulis f. flavicarpa & Brasil - SP & IA 24 & P. edulis f. flavicarpa & Brasil-PR \\
\hline BG19 & Passiflora edulis & Ilha Madeira & IA 10 & P. edulis f. flavicarpa & Brasil-PR \\
\hline BG20 & Passiflora edulis & Marrocos & IA 59 & P. edulis $\mathrm{f}$. flavicarpa & Brasil-PR \\
\hline BG21 & Passiflora edulis & Brasil - PR & IA 58a & P. edulis f. flavicarpa & Brasil-ES \\
\hline BG23 & Passiflora edulis & Brasil - SP & IA 12 & P. edulis $\mathrm{f}$. flavicarpa & Brasil-MG \\
\hline BG24 & Passiflora foetida & Brasil - RJ & IA 43 & P. edulis $\mathrm{f}$. flavicarpa & Brasil-SP \\
\hline BG25 & Passiflora alata & Brasil & IA 91 & P. edulis f. flavicarpa & Brasil-MG \\
\hline BG26 & Passiflora edulis & Chile & IA 29 & P. edulis $\mathrm{f}$. flavicarpa & Brasil-SC \\
\hline BG27 & Passiflora alata & Peru & IA 49 & P. edulis $\mathrm{f}$. flavicarpa & Brasil-ES \\
\hline BG35 & Passiflora sp. & & IA 71 & P. edulis $\mathrm{f}$. flavicarpa & Brasil-PR \\
\hline LM 1 & P. edulis $f$. flavicarpa & Brasil - SP & IA 54 & P. edulis f. flavicarpa & Brasil-PR \\
\hline LM2 & P. edulis f. flavicarpa & Brasil - SP & IA 37 & P. edulis f. flavicarpa & Brasil-PR \\
\hline LM3 & P. edulis $\mathrm{f}$. flavicarpa & Brasil - SP & IA 86 & P. edulis $\mathrm{f}$. flavicarpa & Brasil-MG \\
\hline LM 4 & P. edulis f. flavicarpa & Brasil - PR & IA 11 & P. edulis f. flavicarpa & Brasil-ES \\
\hline LM 5 & P. edulis $\mathrm{f}$. flavicarpa & Brasil - PR & IA $58 \mathrm{~b}$ & P. edulis $\mathrm{f}$. flavicarpa & Brasil-ES \\
\hline LM 6 & $P$. edulis $\mathrm{f}$ flavicarpa & Brasil - PR & IA 62 & P. edulis $\mathrm{f}$ flavicarpa & Brasil-SC \\
\hline LM 7 & P. edulis f. flavicarpa & Brasil-MG & & & \\
\hline
\end{tabular}

LM1=LM180, LM2=LM157,LM3=LM118,LM4=LM156x123 118 x 204,LM5=LM117 x 123, LM6=LM156x 123, LM7=LM123,LM8=LM123 x 54, $\mathrm{LM} 9=\mathrm{LM} 156 \times 180, \mathrm{LM} 10=\mathrm{LM} 87$

TABELA 2- Lista de descritores usados para a caracterização agromorfológica

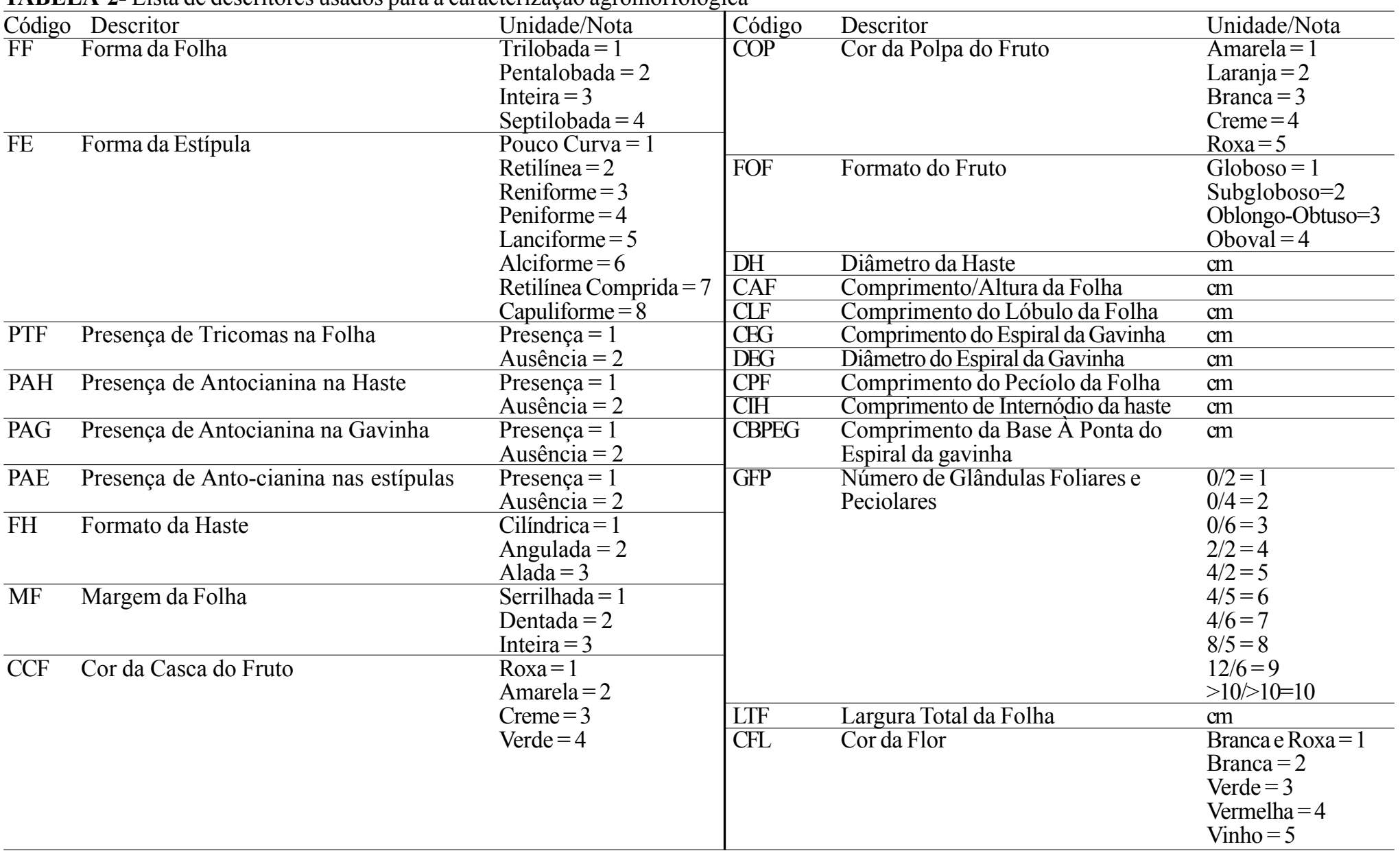




\section{RESULTADOS E DISCUSSÃO}

A partir da matriz de correlação entre os acessos, calcularam-se os componentes principais, possibilitando integrar as múltiplas informações extraídas e identificar quais descritores melhor caracterizaram os materiais estudados. A representação da distribuição das variáveis e a dispersão dos acessos em relação aos dois primeiros componentes principais são apresentadas nas Figuras $1 \mathrm{e} 2$, respectivamente. O primeiro e o segundo componentes representaram 29,6 e $19,4 \%$ do total da variação, respectivamente, enquanto o terceiro representou $12,2 \%$.

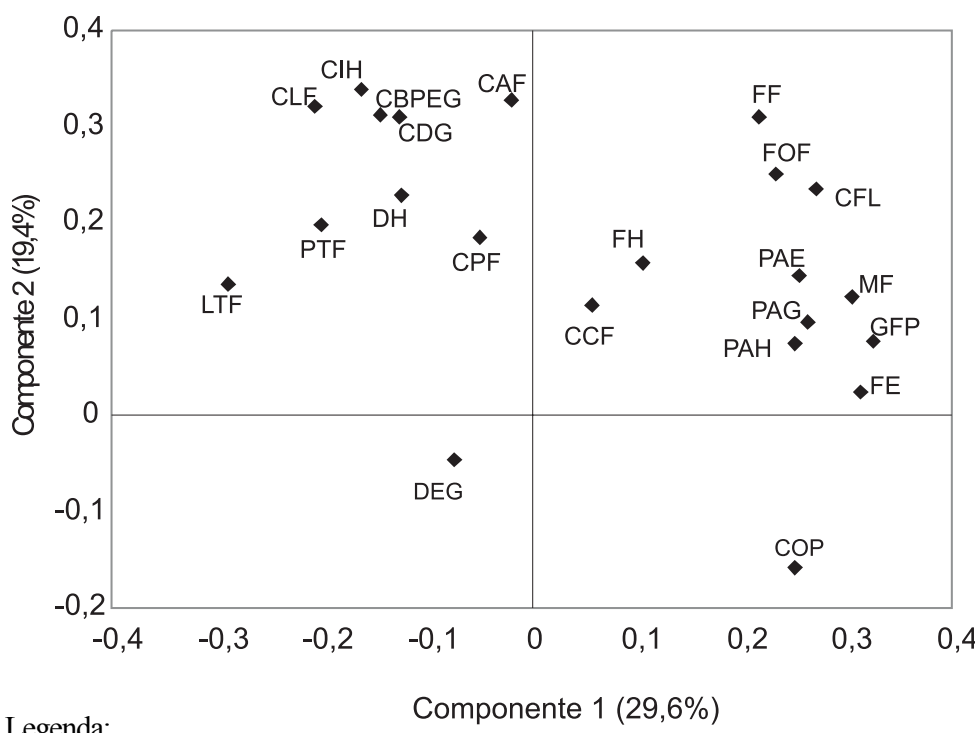

$\mathrm{FF}=$ Forma da Folha $; \mathrm{DH}=$ Diâmetro da Haste $; \mathrm{FE}=$ Forma da Estípula $; \mathrm{CAF}=$ Comprimento/Altura Folha; $\mathrm{PTF}=$ Presença de Tricomas na Folha; $\mathrm{CLF}=\mathrm{Com}-$ primento do Lóbulo da Folha; PAH = Presença de Antocianina nas Hastes; CEG $=$ Comprimento do Espiral Gavinha $; \mathrm{PAG}=$ Presença de Antocianina na Gavinha $\mathrm{DEG}=$ Diâmetro do Espiral Gavinha; $\mathrm{PAE}=$ Presença de Antocianina nas Estípulas $\mathrm{CPF}=$ Comprimento do Pecíolo Folha; $\mathrm{FH}=$ Formato de Haste; $\mathrm{CIH}=$ Comprimento do Internódio Haste; $\mathrm{MF}=$ Margem da Folha; $\mathrm{CBPEG}=$ Comprimento da Base à Ponta do Espiral Gavinha; $\mathrm{CFL}=$ Cor da Flor; $\mathrm{GFP}=$ Número de Glândulas Foliares e Peciolares; $\mathrm{CCF}=$ Cor da Casca do Fruto; $\mathrm{LTF}=$ Largura Total da Folha; COP = Cor da Polpa; FOF = Formato do Fruto

FIGURA 1- Representação de 22 caracteres sobre os componentes 1 e 2 da ACP de 55 acessos de Passiflora spp

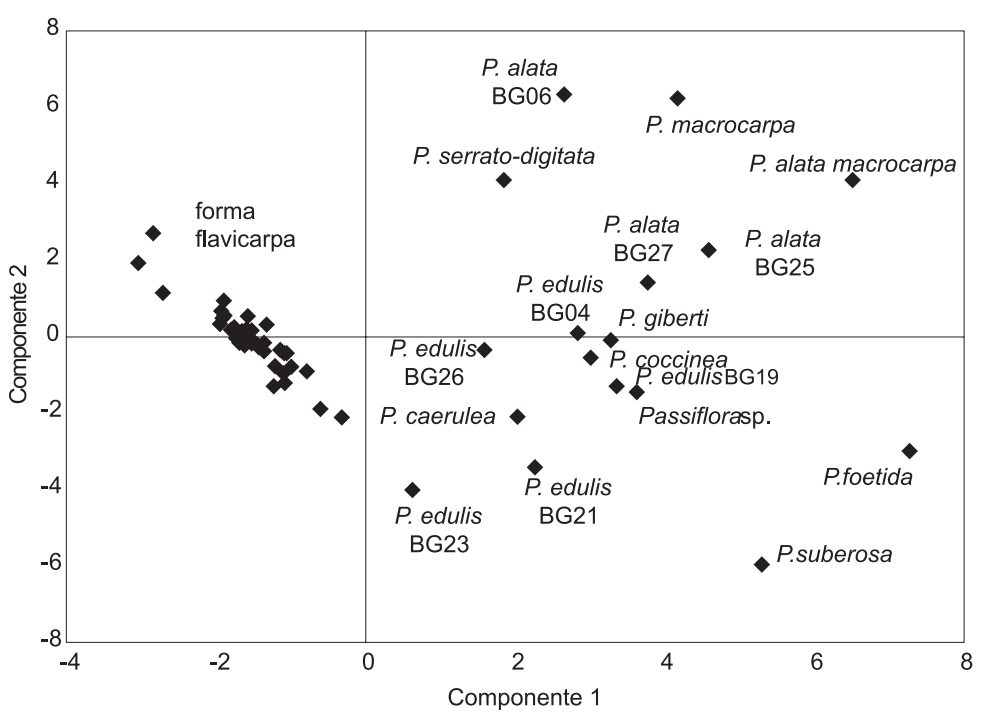

FIGURA 2- Distribuição dos 55 acessos de Passiflora sp. sobre os dois componentes de ACP.

O primeiro componente foi positivamente correlacionado para PAH, PAG, PAE , GFP, MF, FE, CFL, FOF e COP. PTF e LTF foram negativamente correlacionadas para o primeiro componente. $\mathrm{O}$ segundo componente foi positivamente correlacionado para $\mathrm{FF}, \mathrm{CAF}$, CLF, CEG, CIH e CBPEG.

Assim, $P$. coccinea, $P$. foetida, dois acessos de $P$. alata e $P$ alata-macrocarpa, alguns acessos de P. edulis e o acesso Passiflora sp. constituíram um grupo caracterizado por: não apresentar antocianina nas hastes, nas gavinhas nem nas estípulas, número variável de glândulas foliares e peciolares, margem de folhas lisas, estípulas retilíneas, cor de flor do vermelho ao vinho, folhas estreitas, de forma inteira a septilobadas, alguns com presença de tricomas e frutos de forma oblonga-obtusas a oboval, de polpa creme e roxa.

P. macrocarpa e P. alata (BG06) apresentaram folhas inteiras, maior relação comprimento/altura folha, maiores comprimentos de lóbulo de folha, de espiral de gavinha e de internódio de haste e maiores comprimentos da base à ponta da espiral de gavinha. Folha trilobada, menor relação comprimento/altura de folha, menores comprimentos de lóbulo de folha, espiral de gavinhas mais curtas, menores internódios da haste e menores comprimentos da base à ponta do espiral de gavinha foram observados em $P$. caerulea, $P$. suberosa, $P$. edulis (BG21 e BG3) e em 4 acessos da forma flavicarpa (LM157; LM118; LM123, e LM 156x180).

$P$. edulis f. flavicarpa e dois acessos de $P$. edulis estão basicamente distribuídos na parte negativa do primeiro componente e apresentam antocianina nas hastes, gavinhas e estípulas, quatro glândulas foliares e duas peciolares, margem de folha serrilhada, estípulas pouco curvas, flores de cor branca ou branca e roxa, frutos globosos com polpa de cor amarela e laranja. BG18 (P. edulis x P. edulis f. flavicarpa) e BG20 (P. edulis), embora apresentando as características citadas, mostram folhas mais largas, maiores diâmetro de hastes e maiores comprimentos do lóbulo da folha e de internódios de haste, em relação aos demais acessos, e encontram-se na parte positiva do segundo componente.

CPF foi correlacionado positivamente para o terceiro componente. Folhas de pecíolos mais longos são encontradas em $P$. serrato-digitata e $P$. giberti.

No dendrograma (Figura 3), representando os três primeiros componentes (61,2\% da variação), observa-se a estruturação de 4 grandes grupos. O primeiro corresponde à $P$. foetida e $P$. suberosa, que têm alta divergência entre si, assim como com as demais espécies. $P$. foetida, uma das principais espécies para uso medicinal, é a única espécie do estudo que apresentou inúmeras glândulas foliares e peciolares. P. suberosa apresenta apenas duas glândulas peciolares, nenhuma foliar e polpa de fruto roxa, o que a diferencia das demais espécies. O segundo, corresponde ao grupo de $P$. alata e $P$. macrocarpa, que apresentam características folhas inteiras e hastes aladas. Um acesso brasileiro (25) de P. alata é bastante similar ao peruano (27), ambos apresentam antocianina nas estípulas, enquanto os demais do grupo não apresentam esta característica. O terceiro grupo corresponde a $P$. serrato-digitata e P. giberti. No presente estudo, $P$. serrato-digitata é a espécie que apresentou folhas mais largas, septilobadas, maior comprimento do lóbulo e do pecíolo da folha e maior comprimento da base à ponta do espiral de gavinha. $\mathrm{O}$ quarto grupo é composto basicamente por $P$. edulis, com exceção de $P$. coccinea e $P$. caerulea e do acesso Passiflora sp.. P. coccinea e $P$. caerulea alocam-se nesse agrupamento por apresentarem características muito semelhantes a alguns acessos de $P$. edulis, como curta CEG e pequeno $\mathrm{CIH}$.

Considerando-se a nítida separação do grupo $P$. edulis das demais espécies, exceto das espécies $P$. coccinea, $P$. caerulea e Passiflora sp., procurou-se melhor caracterizar estas espécies, realizando-se nova ACP, agora integrando os 46 acessos do grupo. $\mathrm{O}$ descritor CAF, por mostrar-se invariável para o grupo, foi retirado da análise. Os dois primeiros componentes representaram 29,9\% e 18,9\%, respectivamente, do total da variação. A Figura 4 mostra a distribuição dos acessos nos dois primeiros componentes da nova ACP. Observa-se nítida separação de $P$. coccinea de dois grandes grupos, um formado basicamente por $P$. edulis f. flavicarpa e o outro por: $P$. caerulea, P. edulis (BG04, BG19 BG21 BG23 e BG26) e pelo acesso Passiflora sp. 


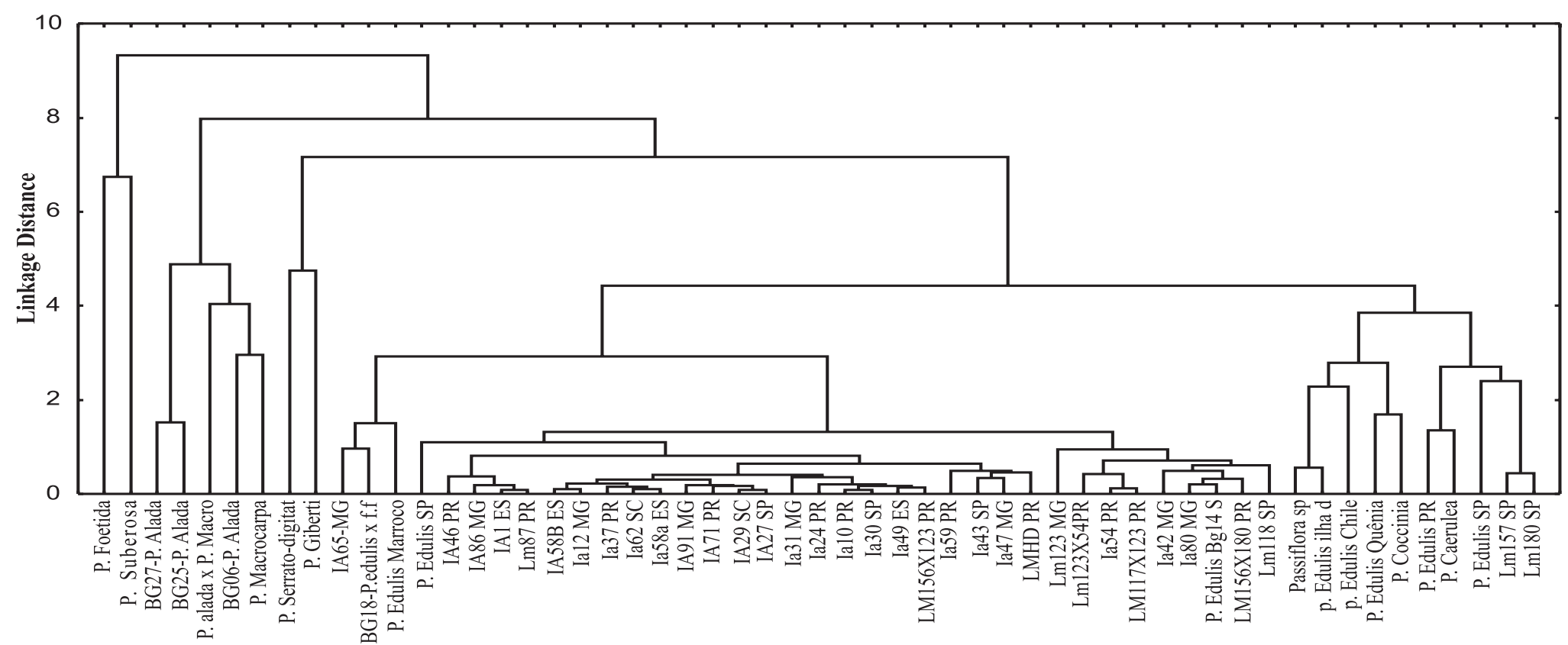

FIGURA 3-Dendrograma dos 55 acessos de Passiflora spp. classificados segundo distâncias Euclidianas pelo método de agregação UPGMA (Unweighted Pair-Group Average). O dendrograma representa os 3 primeiros componentes da ACP.

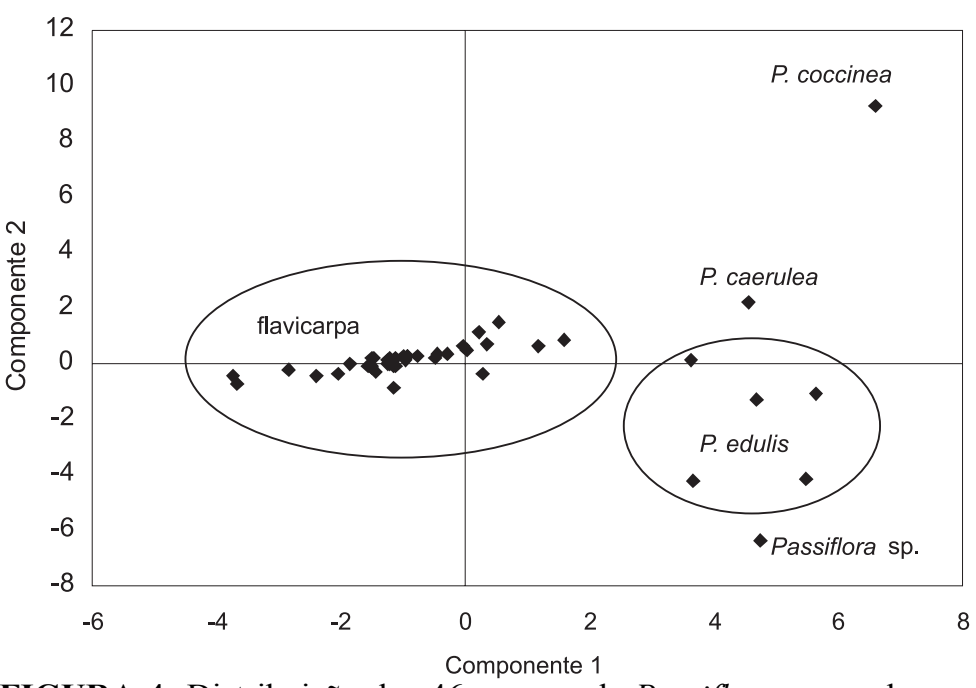

FIGURA 4- Distribuição dos 46 acessos de Passiflora spp. sobre os componentes 1 e 2 da ACP

P. coccinea foi a única espécie, no estudo, que apresentou 8 glândulas foliares e 5 peciolares, menor comprimento de lóbulo da folha e maior diâmetro de espiral de gavinha, cor de flor vermelha e cor da casca do fruto verde, características que a distancia dos demais grupos. Por outro lado, P. coccinea, P. caerulea, Passiflora sp. e os acessos BG04 BG19 BG21 BG23 e BG26 (P. edulis) evidenciaram ampla variação na forma da estípula. $P$. coccinea, Passiflora sp. e BG19 apresentam forma de estípula reniforme. P. caerulea é peniforme. BG04 é lanciforme, BG26 é alciforme, BG23 é retilínea e BG21 é retilínea comprida.

Das espécies estudadas nesta ACP, $P$. coccinea e P. caerulea foram as que apresentaram, respectivamente, maior e menor diâmetro de haste. Por outro lado, maiores diâmetros de haste, maiores $\mathrm{CIH}$ e maiores CBPEG foram encontrados mais na forma flavicarpa do que em $P$. edulis.

Os acessos $P$. edulis não apresentaram antocianina nas hastes, exceto BG09, um material ainda segregante, BG23, uma população selvagem, e BG14, todos brasileiros. Ainda em P. edulis, antocianina nas gavinhas foi encontrada apenas nos acessos provenientes do Quênia (BG04), do Chile (BG26) e da Ilha da Madeira (BG19). Antocianina nas estípulas foi encontrada nos dois últimos acessos (do Chile e da Ilha da Madeira). Por outro lado, a forma flavicarpa apresentou, invariavelmente, presença de antocianina nas hastes, gavinhas e estípulas.

A cor da flor de $P$. edulis (incluindo forma flavicarpa) e do acesso Passiflora sp. é branca e roxa, enquanto em $P$. caerulea, a mais conhecida das Passifloras ornamentais, a flor é azul.

A cor da casca do fruto apresenta-se roxa para $P$. edulis e amarela para $P$. edulis f. flavicarpa e $P$. caerulea, enquanto é verde em $P$. coccinea.

A cor da polpa do fruto para $P$. edulis é laranja, exceto para BG04, que se mostrou amarela. Ao contrário, a forma flavicarpa apresentou polpa de fruto de cor amarela, exceto para os acessos LM180, LM157, LM123, que foi laranja. P. coccinea, P. caerulea e Passiflora sp. apresentaram cor de polpa branca.

O terceiro componente, que representou $10,5 \%$ da variação, correlacionou positivamente MF e negativamente PTF. A distribuição dos acessos no primeiro e no terceiro componentes separa $P$. coccinea e Passiflora sp. dos demais acessos. P. coccinea e P. edulis (BG19) apresentam MF inteira, enquanto no acesso Passiflora sp. é dentada. Em P. caerulea e nos demais acessos $P$. edulis (incluindo forma flavicarpa), ela é serrilhada.

O acesso Passiflora sp. apresentou características bastante intermediárias, ora assemelhando-se à $P$. edulis, ora às espécies $P$. coccinea e $P$. caerulea. Porém, foi o único acesso desse grupo que apresentou tricomas na folha, folhas mais estreitas e de margem dentada. A semelhança de alguns caracteres encontrados nesse acesso, em $P$. coccinea e em $P$. caerulea, leva-nos a supor que Passiflora sp. seja resultado de um possível cruzamento, o que nos leva a sugerir estudos envolvendo hibridação entre estas duas espécies. Porém, é importante ressaltar que tricomas nas folhas também foram encontrados em $P$. suberosa e $P$. foetida.

Com a retirada das espécies $P$. coccinea, $P$. caerulea e do acesso Passiflora sp. da análise, os caracteres PTF, FF e CF tornaram-se monomórficos para $P$. edulis. Estas variáveis, juntamente com PAH, PAG, PAE, GFP, MF, FH, FE, DH, CAF,CLF, DEG, CPF, CBPEG, CCF e FOF, por mostrarem-se pouco discriminantes para detectar divergências dentro da espécie edulis, foram excluídas da nova análise realizada sobre os 43 acessos da espécie.

Apenas quatro descritores foram altamente discriminantes e separaram basicamente os acessos $P$. edulis da forma flavicarpa. São eles CEG, CIH, CCF e COP. A representação e distribuição desses caracteres sobre os dois primeiros componentes de uma nova ACP $(66,4 \% \mathrm{e}$ $18,5 \%$ da variação) estão mostradas, respectivamente, nas Figuras 5 e 6. O primeiro componente correlacionou positivamente os caracteres $\mathrm{CEG}$, $\mathrm{CIH}, \mathrm{CCF}$ e negativamente, $\mathrm{COP}$.

P. edulis apresenta menor comprimento de espiral, internódios de haste mais curtos, casca de frutos de cor roxa, enquanto a forma 
flavicarpa mostra maiores comprimentos de espiral de gavinha, internódios de haste mais longos e casca do fruto amarela. Quanto ao caráter cor da polpa do fruto, a maioria dos acessos da forma flavicarpa apresentou-se na coloração amarela, exceto os lotes de matrizes LM180

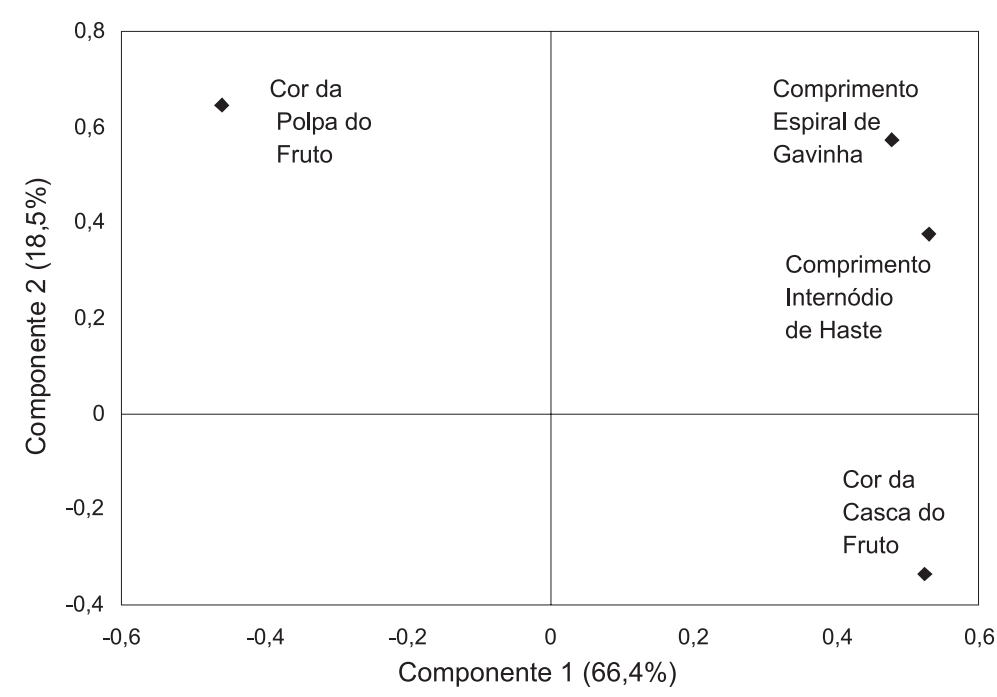

FIGURA 5- Contribuição de 4 caracteres sobre os 2 componentes da ACP de 43 acessos de Passiflora edulis, representando $85 \%$ da variação.

Assim, representando os dois componentes que constituem $85 \%$ do total da variação, é apresentado o dendrograma na Figura 7 , onde se pode observar a estruturação de dois grandes grupos. A pequena divergência encontrada entre os acessos do grupo, à direita, na Figura 7, constituído por $P$. edulis e dois acessos da forma flavicarpa (LM157 e LM180), ambos procedentes de São Paulo-BR, deve-se, sobretudo, à semelhança quanto ao caráter cor de polpa do fruto (laranja). O segundo grupo é constituído predominantemente pelos acessos da e LM157, ambos procedentes de São Paulo-BR e LM123, de Minas Gerais-BR, que apresentaram cor laranja. Para $P$. edulis, este caráter preponderou na cor laranja, exceto para o acesso proveniente do Quênia (BG04), que se apresentou na coloração amarela.

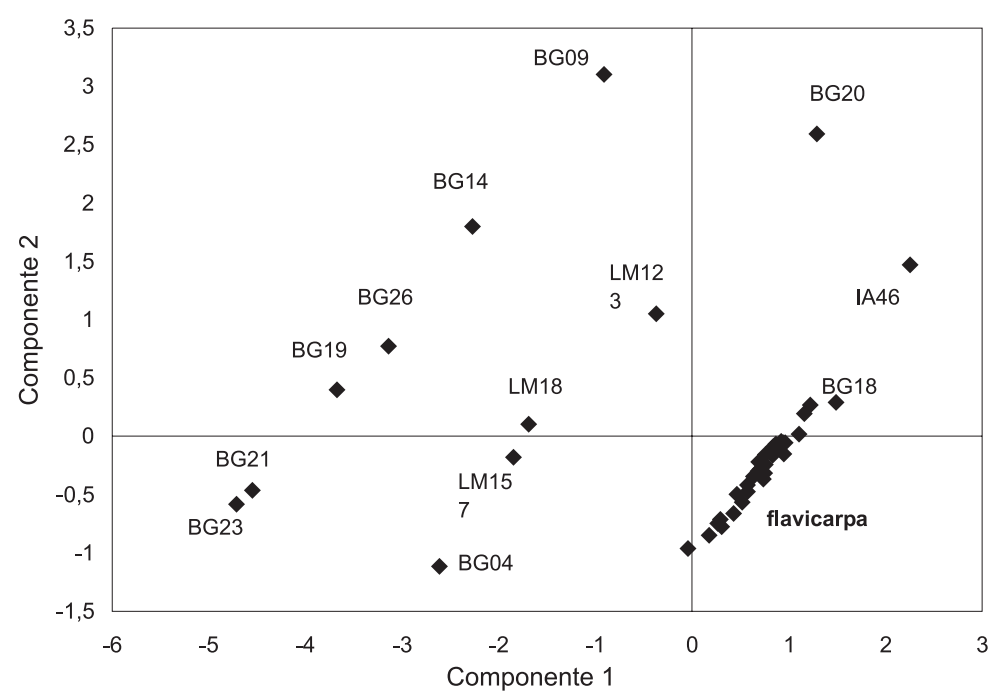

FIGURA 6- Distribuição dos 43 acessos de $P$. edulis nos 2 primeiros componentes da ACP.

forma flavicarpa. O acesso P. edulis (BG20) originário do Marrocos mostrou-se divergente dos demais acessos da espécie, formando, juntamente com um acesso da forma flavicarpa (IA46), um cluster distinto e intermediário. IA46 é o único acesso do grupo flavicarpa que apresentou o maior comprimento de espiral de gavinha, equivalente a um acesso $P$. alata e BG20 apresentou a casca do fruto na cor amarela. De forma isolada dos demais acessos do grupo flavicarpa, encontra-se LM123, de Minas Gerais-BR, mostrando cor laranja para a polpa do fruto.

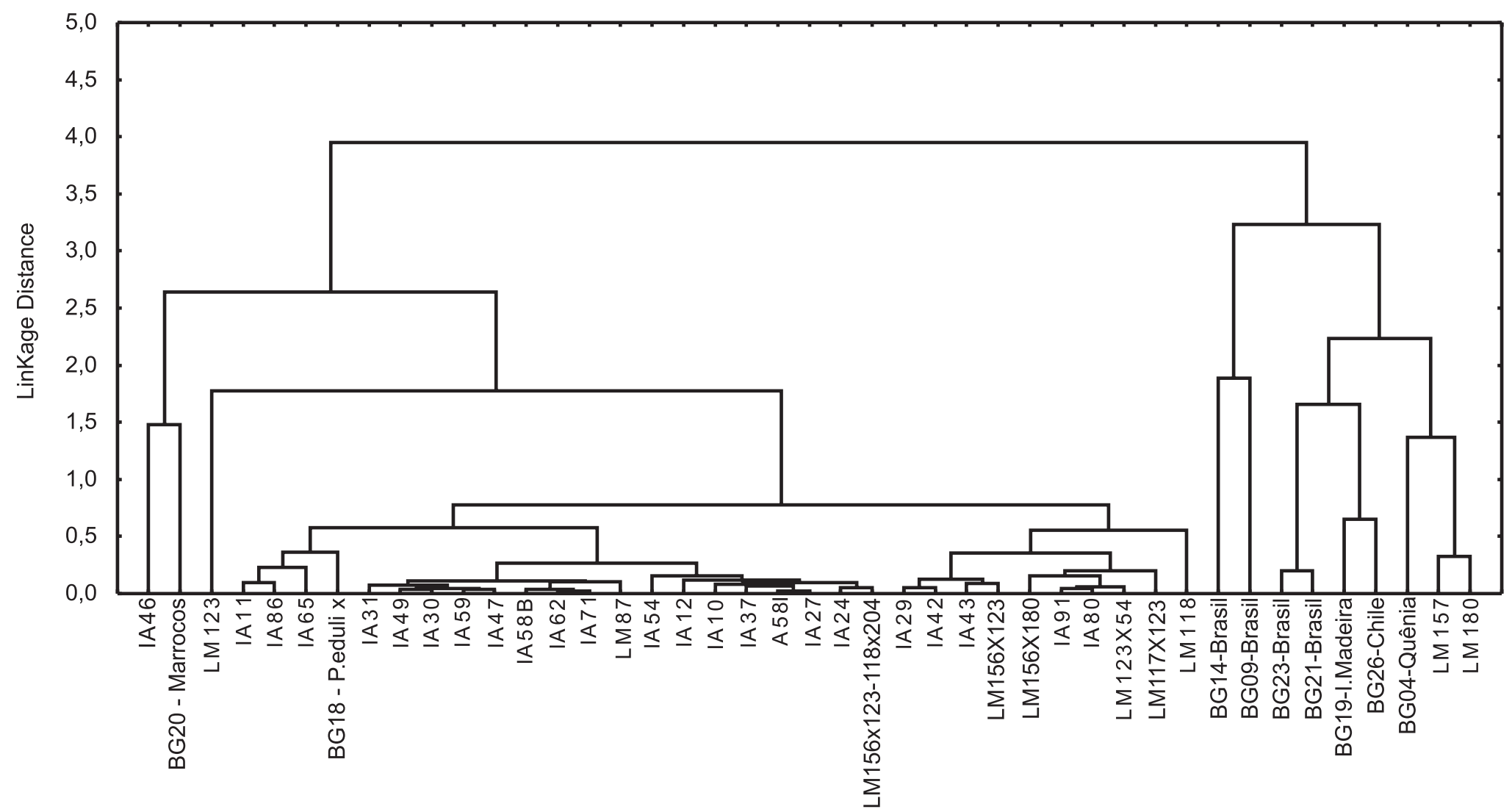

FIGURA 7- Dendrograma dos 43 acessos de Passiflora edulis classificados segundo distâncias Euclidianas pelo método de agregação UPGMA (Unweighted Pair-Group Average). Representa 2 componentes da ACP e 85 \% da variação. 
Uma estruturação baseada em uma pequena divergência genética, associada a uma mesma origem de região geográfica, parece ter sido obtida no subgrupo integrado pelos acessos IA11, do Espírito Santo-BR, IA86, IA65, ambos de Minas Gerais-BR e BG18, de São Paulo. Um pequeno gradiente no comprimento do internódio de haste foi observado neste subgrupo e naquele integrado por: LM118, IA43 (SP-BR), LM117 x 123, LM156 x 123 LM123 x 54, LM156 x 180 (PR-BR), IA80, IA42, IA91 (MG-BR) e IA29 (SC-BR). O gradiente deste caráter, em relação ao grupo precedente, foi levemente superior para o subgrupo constituído por: LM156x123 118x204, IA24, IA27, IA58, IA37, IA10, IA12, IA54, LM87, IA71, IA62, IA58B, IA47, IA59, IA30, IA49 e IA31.

Segundo Nakasone et al. (1967), poucos caracteres do maracujazeiro possuem estudos de herdabilidade, principalmente aqueles de natureza quantitativa. Para esses autores, colorações de gavinha e da casca externa do fruto têm herança monogênica. A coloração do fruto roxa ou amarela é controlada por um par de genes sem dominância, ou seja, $\mathrm{F}_{1}$ apresenta coloração intermediária, roxoavermelhada. No entanto, para Oliveira e Ferreira (1991), a coloração roxa da casca é dominante sobre a coloração amarela. No presente estudo, BG18, originário de uma hibridação de $P$. edulis x $P$. edulis $\mathrm{f}$. flavicarpa efetuada na UNESP (Jaboticabal-SP-BR), apresentou característica coloração amarela da casca, classificando-se no grupo flavicarpa. Em análise RAPD (Crochemore et al., 2002), este acesso agrupou-se, nitidamente, como $P$. edulis. Parece tratar-se de uma dominância incompleta (Ramalho et al., 1997), onde o alelo que codifica a enzima funcional para a presença de antocianina na casca do fruto, a produz em pequena quantidade. Desta forma, no heterozigoto, um único alelo funcional permite que a quantidade de produto final da via metabólica (antocianina) seja também menor do que no homozigoto. Assim, a expressão do heterozigoto é intermediária, roxoavermelhada (Nakasone et al., 1967).

Os caracteres CIH e CEG, embora tenham sido discriminantes, são caracteres quantitativos, podendo estar fortemente ligados ao ambiente. Embora na bibliografia levantada não se tenha relatos sobre a ocorrência natural da forma selvagem de flavicarpa no Brasil, existem informações que indicam a existência destas formas em algumas regiões do Sergipe e de Santa Catarina. Por outro lado, formas mutantes amarelas são encontradas regularmente na Austrália (Vanderplank, 1996). Apenas após a avaliação destes materiais poder-se-á chegar à conclusão de que se trata de uma outra espécie ou de apenas uma diferenciação agronômica, como já reportado por outros autores (Piza, 1966; Martin \& Nakasone, 1970),

A avaliação dos acessos em apenas um ambiente limita inferências mais precisas sobre a expressão do potencial genético dos mesmos. Sugere-se, portanto, a inclusão de novos ambientes e ampliação de acessos nos próximos estudos, utilizando-se também de um maior número de descritores, principalmente aqueles relacionados à produção, incrementando-se estudos relacionados à genética de caracteres quantitativos.

\section{CONCLUSÕES}

Ampla divergência genética foi encontrada entre as espécies, e importante variação foi observada entre os acessos de $P$. edulis e do grupo $P$. alata/P.macrocarpa. P. caerulea apresentou menor diâmetro de haste em relação aos demais acessos. O maior número de glândulas peciolares foi encontrado em $P$. foetida. Este, juntamente com $P$. suberosa, e Passiflora sp. foram os únicos acessos que mostraram tricomas nas folhas. A forma de estípula capuliforme foi encontrada exclusivamente em P. giberti. Comprimento de gavinha e de internódio de haste, cor da casca e da polpa do fruto foram altamente discriminantes e separaram basicamente $P$. edulis da forma flavicarpa. $\mathrm{O}$ acesso $P$. edulis originário do Marrocos mostrou-se divergente dos demais acessos da espécie, formando, juntamente com um acesso da forma flavicarpa, um cluster distinto e intermediário. Apesar de os descritores utilizados mostrarem grande variação dentro do grupo $P$. edulis, limitada variabilidade foi encontrada dentro do grupo flavicarpa.

\section{REFERÊNCIAS BIBLIOGRÁFICAS}

BEAL, P.R. Hybridization of Passiflora edulis Sims and P. edulis f. flavicarpa Degener. Queensland Journal of Agricultural and Animal Science, Brisbane, v.32,n.1,p.101-111, 1975.

BRUCKNER, C. Perspectivas do melhoramento genético do maracujazeiro. Maracujá: temas selecionados, Porto Alegre: Cinco Continentes Editora, 1997. p. 25-46.

CROCHEMORE, M.L.; MOLINARI, H.B.; VIEIRA, L.G.E. Genetic diversity in passion fruit (Passiflora spp.) evaluated by RAPD markers. Brazilian Archives of Biology and Technology. 2002 (in press).

HOEHNE, F.C. Frutas indígenas. São Paulo: Instituto de Botânica, 1946. $88 \mathrm{p}$.

LOPES, S.C. Citogenética do maracujá, Passiflora spp. In: SÃO JOSÉ, A.R. A cultura do maracujá no Brasil. Jaboticabal: FUNEP, 1991.p. 201-209

MARTIN, F. W.; NAKASONE, H. Y. The edible species of Passiflora. Economic Botany, Bronx, n. 24, p. 333-43, 1970.

NAKASONE, H.Y.; HIRANO, R.; ITO, P. Preliminary observations on the inhetitance of several factores in the passion fruit Passiflora edulis and forma flavicarpa. Hawaii: Agric. Exp. Sta., 1967. 11p. (Tec. Progr. Rep., 161).

OLIVEIRA, J.C.; FERREIRA, F.R. Melhoramento Genético do Maracujazeiro. In: SÃO JOSÉ, A.R.; FERREIRA, F.R.; VAZ, R.L. (Coord.), A cultura do maracujá no Brasil. Jaboticabal: FUNEP, 1991.p. 211-239.

RAMALHO, M.; SANTOS, J.B.; PINTO, C.B. Genética na Agropecuária, 6.ed. São Paulo: Globo, 1997.359p.

PIZAJR., C. DE T. A cultura do maracujá. São Paulo: Secretaria da Agricultura, 1966. 102p. (Boletim Técnico 5).

SOUSA, J.S.I; MELETTI, L.M.M. Maracujá: espécies, variedades, cultivo. Piracicaba: FEALq, 1997. 179p.

VANDERPLANK, J. Passion flowers. 2.ed. Massachusetts: Ed. Cambridge, 1996.224p. 